\title{
Study of Beta-Catenin Expression: In Endometrial Hyperplasia and Carcinoma
}

\author{
Sagarika Sarkar ${ }^{1 *}$, Ranu Sarkar ${ }^{2}$, Binny Khandakar ${ }^{2}$, Moumita Maiti ${ }^{2}$ \\ Neepamanjari Barman ${ }^{2}$ and Chandana Das $^{3}$ \\ ${ }^{1}$ Department of Pathology, East Point College of Medical Sciences and Research Centre, Bangalore, India \\ ${ }^{2}$ Department of Pathology, Nilratan Sircar Medical College and Hospital, Kolkata, India \\ ${ }^{3}$ Department of Obstetrics and Gynecology, Nilratan Sircar Medical College and Hospital, Kolkata, India
}

\section{ABSTRACT}

Background: Beta-catenin is normally expressed in the membrane and cytoplasm of endometrial glandular cells. Aberrations in beta-catenin expression can predict progression of endometrial hyperplasia to endometrial carcinoma. Nuclear expression of beta-catenin correlates with the increasing histological grade of endometrial cancer.

Methods: 51 cases were included in our study. The patients presented with clinical and/or radiological evidence of probable endometrial disease. Formalin fixed paraffin embedded tissues were stained with hematoxylin and eosin followed by histological diagnosis and exact categorisation. Immunostaining with anti-beta-catenin monoclonal antibody carried out on these endometrial biopsies.

Result: Statistically significant association was seen between nuclear positivity of beta-catenin in the endometrial glandular cells with increasing severity of endometrial pathology $(\mathrm{P}<0.001)$. Atypical hyperplasia and endometrial carcinoma cases showed nuclear beta-catenin positivity. Nuclear expression of beta-catenin also correlated with advanced FIGO stage of endometrial carcinomas. $67 \%$ of endometrial carcinoma of FIGO stage III demonstrated nuclear localization of beta-catenin. A statistically significant association was noted between the intensity of beta-catenin expression and the histological diagnosis $(\mathrm{P}<0.001)$. There was also a statistically significant association between percentage of endometrial glandular cells showing membranous and cytoplasmic positivity and the endometrial pathology $(\mathrm{P}=0.038)$.

Conclusion: Variations in beta-catenin expression play an important role in endometrial pathology and it is a relatively early event during the endometrial hyperplasia-carcinoma sequence. Alterations in beta-catenin expression in atypical endometrial hyperplasia and in increasing grades of endometrial cancers can be used as a predictive as well as a prognostic indicator.

Keywords: Beta-Catenin; Proliferative Endometrium; Atypical Hyperplasia; Endometrial Carcinoma

\section{Introduction}

Endometrial hyperplasia, one of the common endometrial pathologies, is defined as proliferation of glands of irregular size and shape and an increase in the gland/stroma ratio compared to proliferative endometrium. ${ }^{[1]}$ It often precedes the development of endometrial carcinoma, usually the endometrioid variant (type I cancer). Serous carcinoma (type II cancer) is the prototypic endometrial carcinoma that is unrelated to estrogenic stimulation or hyperplasia. Type I cancer frequently arises from atypical endometrial hyperplasia, whereas type II cancer arises from a precursor lesion termed as endometrial intraepithelial carcinoma (EIC). ${ }^{[2]}$ Current taxonomy classifies endometrial hyperplasia into two categories depending on presence or absence of atypia. ${ }^{[3]}$ In 1985 , Kurman et al evaluated that the risk of progression to carcinoma was $23 \%$ for atypical endometrial hyperplasia, while only $2 \%$ for non-atypical hyperplasia. ${ }^{[3]}$ Endometrial biopsy is an imperative tool to diagnose endometrial premalignant and malignant lesions.
Beta-catenin, a cadherin associated protein, is a proto oncogene that has a dual role in cell adhesion and transcriptional activation. It binds to intracytoplasmic domains of cadherins and combines with the actin cytoskeleton via alpha-catenin to form cell-cell adherens junctions. ${ }^{[4-6]}$ Disruption of this cell-cell adhesion in malignant cells is responsible for the enhanced migration of tumor cells, leading to invasion and wide spread metastases. Beta-catenin also acts as a nuclear transcription factor as it co-activates the lymphoid enhancer factor (LEF)/T-cell factor (TCF) family of DNA-binding proteins. Activation of Wnt signalling involves the inhibition of beta-catenin degradation by proteasomes, resulting in its cytoplasmic accumulation followed by nuclear localization leading to transcriptional activation of LEF/TCF target genes. ${ }^{[7-8]}$

Endometrial glandular cells show cytoplasmic and membranous expression of beta-catenin. Cytoplasmic beta-catenin is more strongly expressed in the basalis than functionalis during the menstrual cycle. ${ }^{[9]}$ Nuclear 
accumulation of beta-catenin as a result of beta-catenin gene mutation is mainly described in atypical endometrial hyperplasia and endometrial carcinoma. ${ }^{[8-10]}$

The current study aims to analyse beta-catenin expression in proliferative endometrium, endometrial hyperplasia and endometrial carcinoma.

\section{Materials and Methods}

The study was conducted in the Department of Pathology in collaboration with the Department of Obstetrics and Gynaecology, Nilratan Sircar Medical College and Hospital, Kolkata from February 2015 to July 2016. The study included patients attending the Department of Gynecology and Obstetrics, with clinical and/or radiological suspicion of endometrial pathologies. Patients with history and/or signs and symptoms of cervical and adenexal pathologies were excluded. The sample size was 51 which included proliferative endometrium $(n=6)$, endometrial hyperplasia $(\mathrm{n}=19)$ [hyperplasia without atypia: 14 and atypical hyperplasia: 5] and endometrial carcinoma $(\mathrm{n}=26)$ [endometrioid carcinoma: 24 and serous carcinoma: 2]. It was an observational and noninterventional study. Clinical parameters were analyzed followed by morphological diagnosis and categorization of endometrial biopsies ( 37 resected specimens and 14 small tissue biopsies). The tissues were fixed in $10 \%$ formalin, sections cut at 4 micron thickness and stained with hematoxylin and eosin. The following points were noted: phase of menstrual cycle; hyperplastic changes; presence or absence of atypia; histopathological diagnosis, subtyping, grading and pathological staging of endometrial carcinoma. Immunohistochemistry was performed using anti-beta-catenin monoclonal antibody taking normal colonic tissue as control. The following parameters were assessed: location of beta-catenin, percentage of cells showing positive membranous and cytoplasmic staining and the intensity of beta-catenin expression. Statistical analysis of data was carried out using SPSS version 20.

\section{Result}

Total 51 cases were included in the study: proliferative endometrium $(n=6)$, endometrial hyperplasia $(n=19)$ [14 cases of hyperplasia without atypia and 5 cases of atypical hyperplasia] and endometrial carcinoma $(n=26)$ [ 24 cases of endometrioid carcinoma and 2 cases of serous carcinoma].

Epidemiology \& Clinical Parameters: The age range of the study was $31-70$ years. $53 \%$ of the cases diagnosed as endometrial hyperplasia were 31-40 years of age. However, $46 \%$ of the cases of endometrial carcinoma was found to occur in the $5^{\text {th }}$ and $6^{\text {th }}$ decades of life and $42 \%$ of endometrial carcinoma was found in the $6^{\text {th }}$ to $7^{\text {th }}$ decades. [Table 1].

Cases of endometrial hyperplasia were mostly second $(\sim 37 \%)$ and third $(\sim 47 \%)$ gravida while those of endometrial carcinoma were second $(\sim 39 \%)$, third $(\sim 46 \%)$ and fourth $(\sim 15 \%)$ gravida. Out of all cases of endometrial hyperplasia, $68 \%$ were pre-menopausal while all the cases $(100 \%)$ of endometrial carcinoma cases were postmenopausal [Graph 1].

Distribution of Stage of endometrial cancer cases: 14/24 $(\sim 58 \%)$ of endometrioid carcinoma cases were FIGO stage I, $3 / 24(\sim 12.5 \%)$ were FIGO stage II and 7/24 ( 29.7\%) were FIGO stage III; $2 / 2(100 \%)$ cases of serous carcinoma were FIGO stage III [Graph 2].

Analysis of beta-catenin expression in glandular cells of normal endometrium, endometrial hyperplasia and carcinoma

Location of Beta-Catenin Expression: Beta catenin was found to be expressed in three different locations, the cytoplasmic membrane (M), cytoplasm (C) and nucleus $(\mathrm{N})$.

In Normal Endometrium vs Endometrial Hyperplasia vs Carcinoma: All 6 cases of proliferative endometrium (histologically normal/unremarkable) showed localization of beta-catenin in the membrane and cytoplasm only and $>90 \%$ cells showed beta catenin expression [table 2]. $42 \%$ cases with endometrial pathology [hyperplasia and carcinoma] showed membrane and cytoplasmic localization of beta-catenin [in $>90 \%$ cells], the remaining $58 \%$ cases also showed nuclear expression, with relatively reduced ( $\leq 90 \%$ cells positive) cytoplasmic and membrane expression. The intensity of the staining has been analysed too, which is described subsequently. We observed statistically significant difference in the location of beta catenin expression between normal endometrium, endometrial hyperplasia and endometrial cancer, with presence of nuclear beta catenin in atypical endometrial hyperplasia [1] and carcinoma [12] $(P<0.001)$. Majority [18/19] of endometrial hyperplasia [ $\mathrm{n}=19]$, including non-atypical and atypical, showed $\mathrm{M}+\mathrm{C}$ expression [Table 2-3].

In Endometrial carcinoma [ $\mathbf{n}=\mathbf{2 6}]$ : comparison with grade \& stage: 14/26 ( 54\%) of endometrial carcinoma showed membrane and cytoplasmic positivity of betacatenin, while $12 / 26$ cases $(\sim 46 \%)$ showed nuclear localization of beta-catenin, out of which all grade 3 endometrioid carcinoma $(n=6)$ and serous carcinoma $(n=2)$ showed nuclear expression of beta-catenin. [Table 3] 
$35 \%$ of endometrial carcinoma cases of FIGO stage I and II showed nuclear localization of beta-catenin while majority, $67 \%$, of endometrial carcinoma cases of FIGO stage III demonstrated nuclear localization of betacatenin. [Table 4]

Intensity of Beta-Catenin Expression: For membrane and cytoplasmic staining, the intensity of staining/ expression of beta-catenin were sub-classified in to: 1+ weak/faint, $2+$ strong. The intensity of beta-catenin in the proliferative endometrium was $2+(\mathrm{M})$ and $2+(\mathrm{C})$. We found varied intensity of membrane (M) and cytoplasmic (C) staining which included the following four combinations: $1+(\mathrm{C}) 2+(\mathrm{M}) ; 2+(\mathrm{C}) 2+(\mathrm{M}) ; 2+(\mathrm{C}) 1+(\mathrm{M}) ; 2+\mathrm{C} 1+(\mathrm{M})$
N. We observed that there was gradual diminution of intensity of membrane staining and increase in intensity of cytoplasmic staining with the appearance of nuclear expression of beta-catenin from normal to hyperplasia and from hyperplasia without atypia to atypical hyperplasia, and from hyperplasia to carcinoma. Furthermore, all cases of endometrioid carcinoma grade 3 and serous carcinoma showed low membrane $[1+(\mathrm{M})]$ and a stronger cytoplasmic $[2+(\mathrm{C})]$ staining/expression along with nuclear positivity/ expression of beta-catenin. Greater nuclear expression in carcinomas has already been described in the previous section. The difference in staining intensity across the various groups was statistically significant (Chi-square; $P$ $<0.001)$ [Table 5].

Table 1: Distribution of the cases according to age [n=51].

\begin{tabular}{|c|c|c|c|}
\hline Age interval (years) & $\begin{array}{l}\text { Proliferative endometrium } \\
\qquad[\mathrm{n}=6(100 \%)]\end{array}$ & $\begin{array}{l}\text { Endometrial hyperplasia } \\
\qquad[n=19(100 \%)]\end{array}$ & $\begin{array}{l}\text { Endometrial Carcinoma } \\
{[\mathrm{n}=26(100 \%)]}\end{array}$ \\
\hline $31-40$ & $6(100 \%)$ & $10(52.6 \%)$ & $0(0 \%)$ \\
\hline $41-50$ & $0(0 \%)$ & $7(36.8 \%)$ & $3(11.5 \%)$ \\
\hline $51-60$ & $0(0 \%)$ & $2(10.5 \%)$ & $12(46.2 \%)$ \\
\hline $61-70$ & $0(0 \%)$ & $0(0 \%)$ & $11(42.3 \%)$ \\
\hline Total $[n=51]$ & $6(100 \%)$ & $19(100 \%)$ & $26(100 \%)$ \\
\hline
\end{tabular}

Table 2: Extent of beta-catenin expression in membrane and cytoplasm [n=51]

\begin{tabular}{|l|c|c|}
\hline Histological diagnosis & Cases with $>90 \%$ cells positive & Cases with $\leq \mathbf{9 0 \%}$ cells positive \\
\hline Proliferative endometrium [n=6 (100\%)] & $6(100 \%)$ & $0(0 \%)$ \\
\hline Endometrial hyperplasia without atypia $[\mathrm{n}=14(100 \%)]$ & $7(50 \%)$ & $7(50 \%)$ \\
\hline Endometrial hyperplasia with atypia $[\mathrm{n}=5(100 \%)]$ & $1(20 \%)$ & $4(80 \%)$ \\
\hline Endometrial carcinoma [( $=26(100 \%)]$ & $11(42.3 \%)$ & $15(57.7 \%)$ \\
\hline Total $[\mathrm{n}=51]$ & $\mathbf{2 6}$ & $\mathbf{2 5}$ \\
\hline
\end{tabular}

Table 3: Localization of beta-catenin in proliferative endometrium, endometrial hyperplasia and endometrial carcinoma [n=51]

\begin{tabular}{|c|c|c|}
\hline Histological diagnosis $(n=51)$ & $M+C$ & $M+C+N$ \\
\hline Proliferative endometrium [n=6 (100\%)] & $6(100 \%)$ & $0(0 \%)$ \\
\hline Endometrial hyperplasia without atypia [n=14 (100\%)] & $14(100 \%)$ & $0(0 \%)$ \\
\hline Endometrial hyperplasia with atypia [n=5 (100\%)] & $4(80 \%)$ & $1(20 \%)$ \\
\hline Endometrioid carcinoma Grade 1 [n=7 (100\%)] & $7(100 \%)$ & $0(0 \%)$ \\
\hline Endometrioid carcinoma Grade 2 [n=10 (100\%)] & $7(70 \%)$ & $3(30 \%)$ \\
\hline Endometrioid carcinoma Grade 3 [n=7 (100\%)] & $0(0 \%)$ & $7(100 \%)$ \\
\hline Serous Carcinoma $[n=2(100 \%)]$ & $0(0 \%)$ & $2(100 \%)$ \\
\hline Total $[n=51]$ & 38 & 13 \\
\hline
\end{tabular}

Table 4: Correlation of localization of beta-catenin with FIGO stage of endometrial carcinomas [n=26].

\begin{tabular}{|c|c|c|}
\hline FIGO Stage & $\mathbf{M}+\mathbf{C}$ & $\mathbf{M}+\mathbf{C}+\mathbf{N}$ \\
\hline I and II $[\mathrm{n}=17(100 \%)]$ & $11(64.7 \%)$ & $6(35.3 \%)$ \\
\hline III $[\mathrm{n}=9(100 \%)]$ & $3(33.3 \%)$ & $6(66.7 \%)$ \\
\hline Total $(\mathbf{n = 2 6 )}$ & 14 & 12 \\
\hline
\end{tabular}


Table 5: Intensity of beta-catenin expression in proliferative endometrium, endometrial hyperplasia with and without atypia and endometrial carcinoma $[\mathrm{n}=51]$.

\begin{tabular}{|c|c|c|c|c|}
\hline Histological diagnosis [ $n=51]$ & $2+(M), 1+(C)$ & $2+(M), 2+(C)$ & $1+(M), 2+(C)$ & I+(M), $2+(C), N$ \\
\hline Proliferative endometrium [n=6 (100\%)] & $0(0 \%)$ & $6(100 \%)$ & $0(0 \%)$ & $0(0 \%)$ \\
\hline Endometrial hyperplasia without atypia [n=14 (100\%)] & $7(50 \%)$ & $4(28.6 \%)$ & $3(21.4 \%)$ & $0(0 \%)$ \\
\hline Endometrial hyperplasia with atypia [n=5 (100\%)] & $0(0 \%)$ & $0(0 \%)$ & $4(80 \%)$ & $1(20 \%)$ \\
\hline Endometrial carcinoma [n=26 (100\%)] & $0(0 \%)$ & $1(3.8 \%)$ & $13(50 \%)$ & $12(46.2)$ \\
\hline Total $(n=51)$ & 7 & 11 & 20 & 13 \\
\hline
\end{tabular}

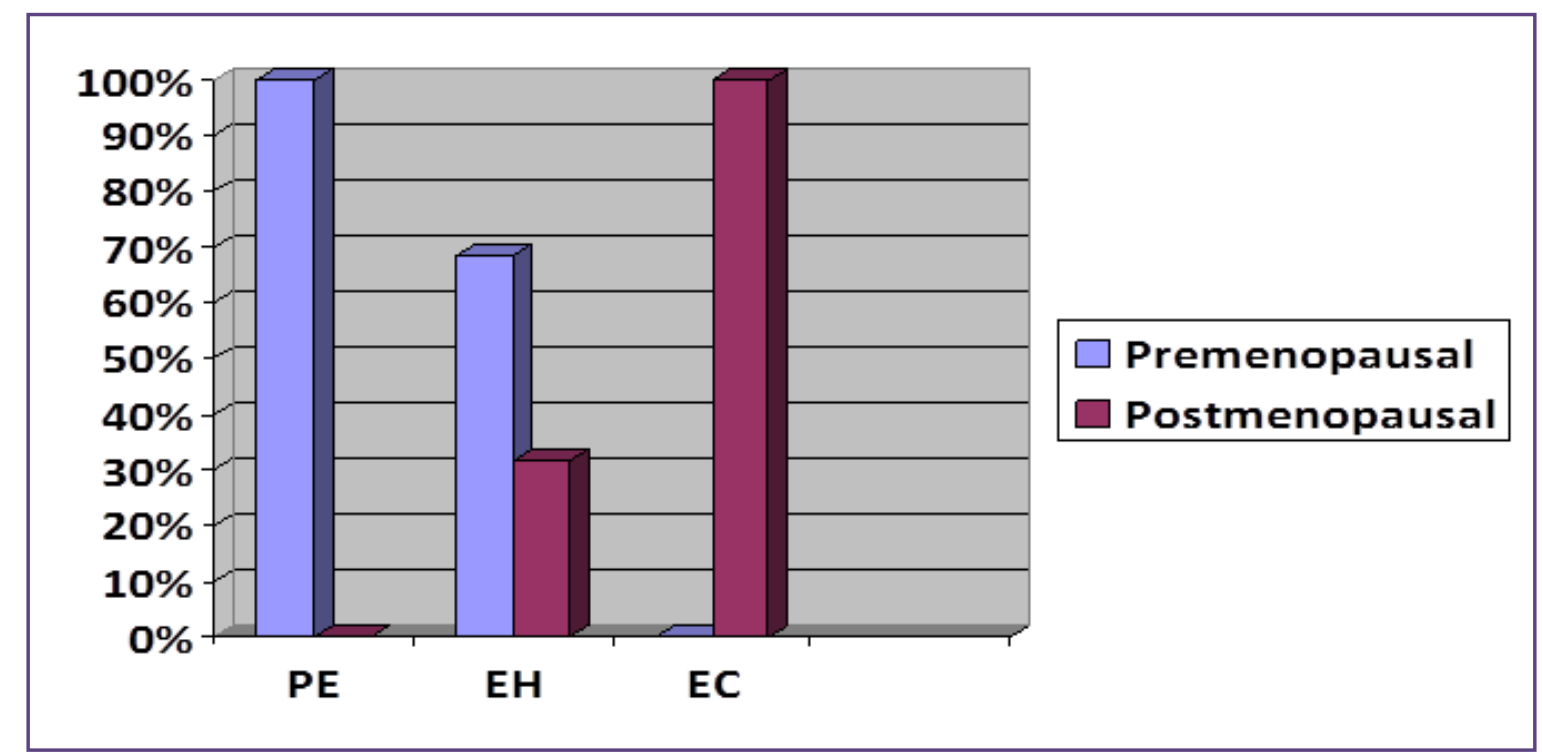

Graph 1: Distribution of cases according to menstrual status (PE- proliferative endometrium, EHendometrial hyperplasia, EC- endometrial carcinoma).

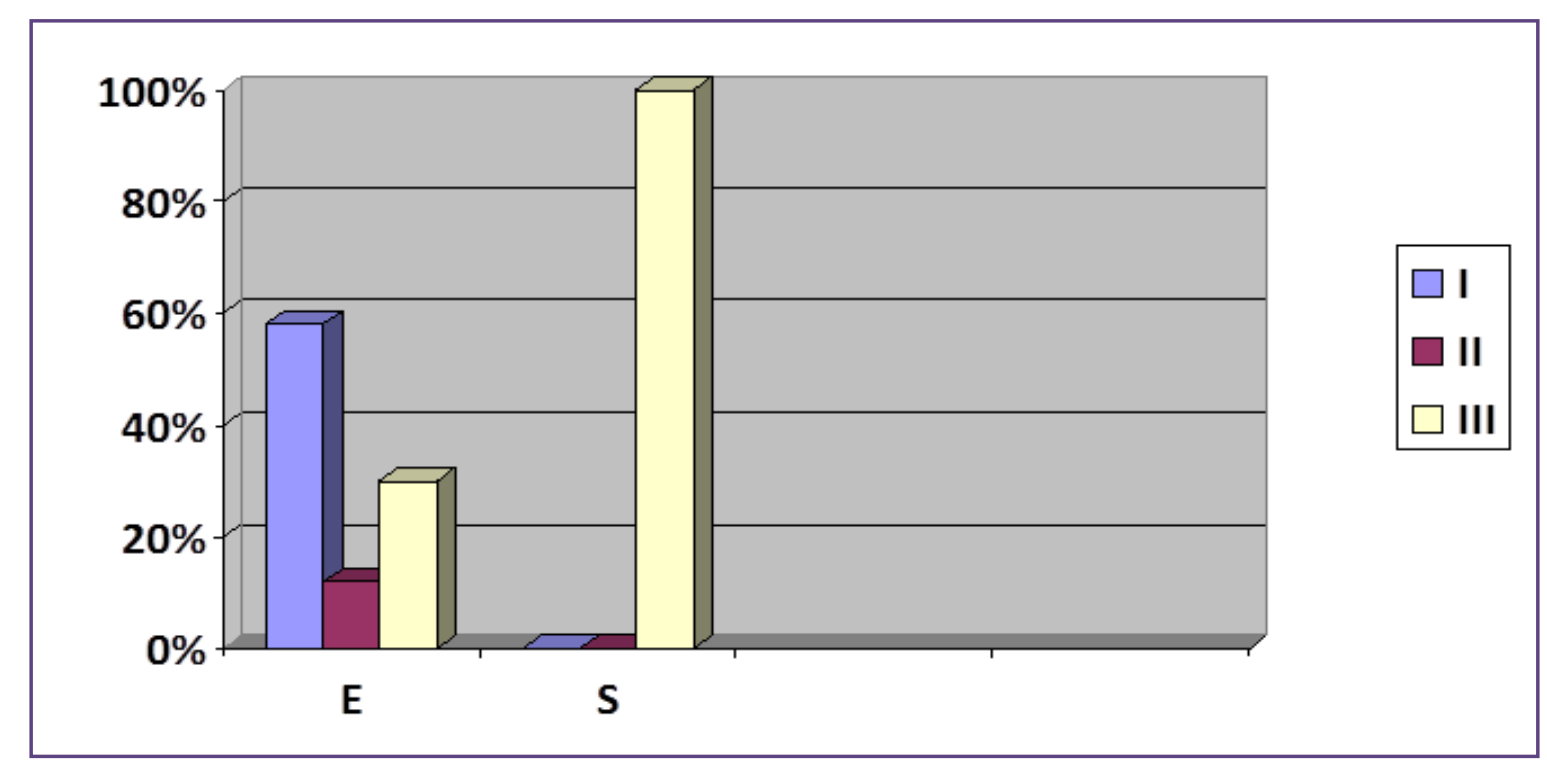

Graph 2: Distribution of type of endometrial carcinoma according to FIGO stage (Endometrioid carcinomaE, Serous carcinoma- S) 


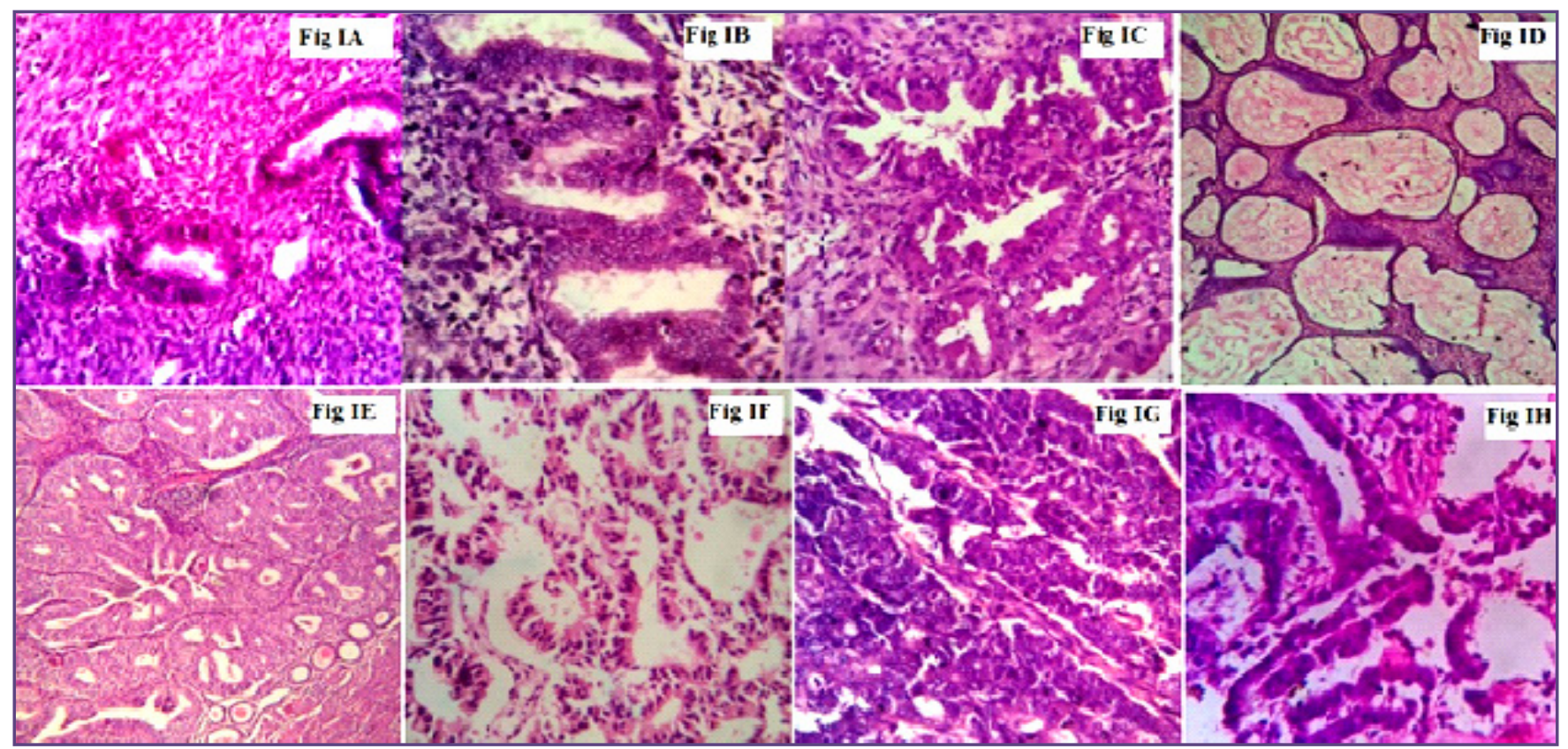

Fig. I: Photomicrographs Fig IA: Proliferative endometrium (H\&E, X400); Fig IB: Endometrial hyperplasia without atypia (H\&E, X400); Fig IC: Atypical endometrial hyperplasia (H\&E, X400); Fig ID: Atypical hyperplasia in an endometrial polyp (H\&E, X100); Fig IE: Endometrioid carcinoma grade 1 (H\&E, X100); Fig IF: Endometrioid carcinoma grade 2 (H\&E, X400); Fig IG: Endometrioid carcinoma grade 3 (H\&E, X400); Fig IH: Serous endometrial carcinoma (H\&E, X400).

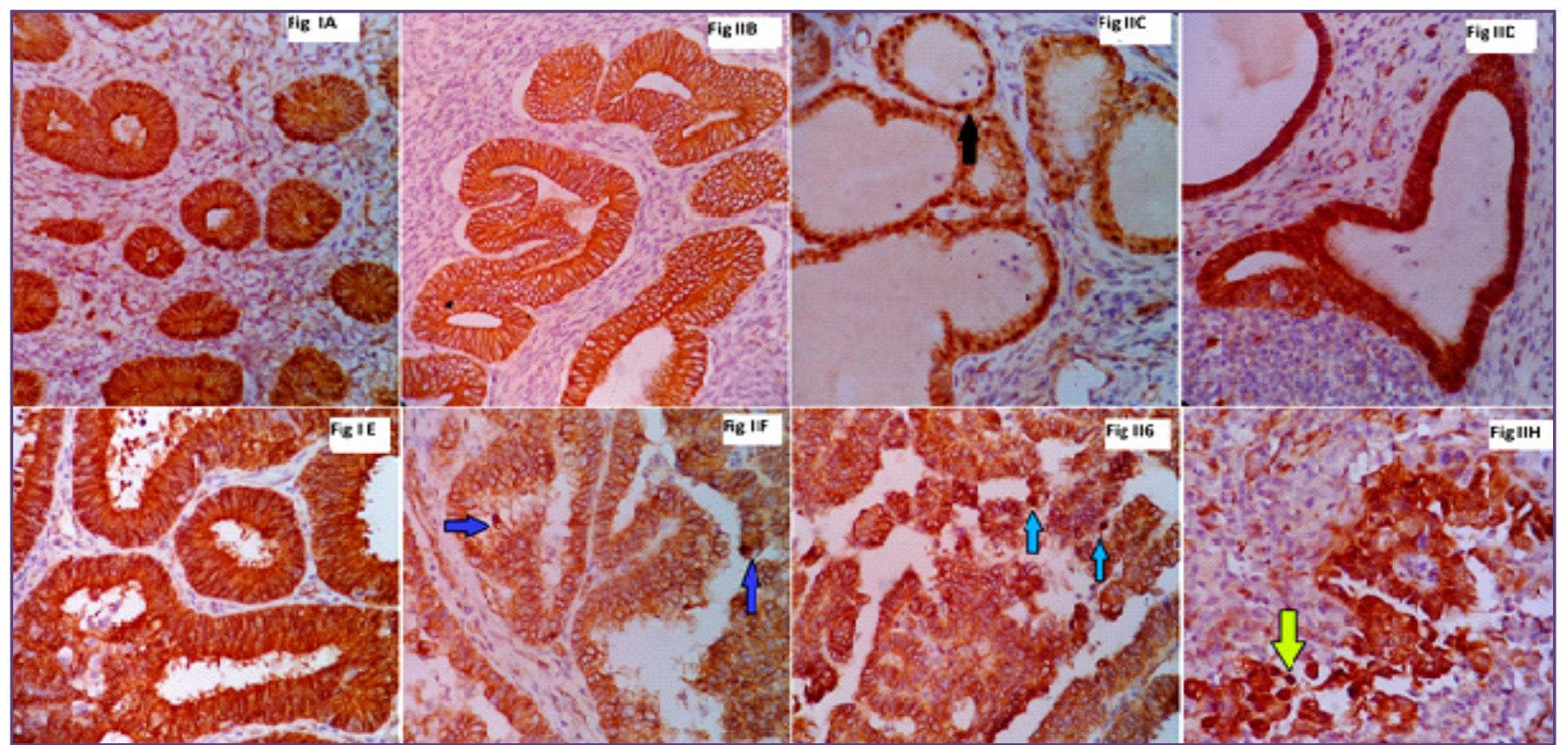

Figure II: Immunohistochemistry with beta-catenin: Fig IIA: Proliferative endometrium- strong membranous and cytoplasmic positivity (DAB chromogen, X400); Fig IIB: Endometrial hyperplasia without atypia- Moderate membranous and cytoplasmic staining intensity (DAB chromogen, X400); Fig IIC: Atypical endometrial hyperplasia- Nuclear expression of beta-catenin (black arrow); Fig IID: Atypical endometrial hyperplasia- Stronger cytoplasmic but weaker membranous staining (DAB chromogen, X400); Fig IIE: Endometrioid carcinoma grade 1- Weaker membranous and moderate cytoplasmic positivity (DAB chromogen, X400); Fig IIF: Endometrioid carcinoma grade 2- Weaker membranous and cytoplasmic staining with nuclear expression of beta-catenin (blue arrow) (DAB chromogen, X400); Fig IIG: Endometrioid carcinoma grade 3- Nuclear localization (Sky blue arrows) (DAB chromogen, X400); Fig IIH: Serous endometrial carcinoma- Increased nuclear expression (light green arrow) and diminished membranous positivity (DAB chromogen, $\mathrm{X400}$ ) 


\section{Discussion}

Beta-catenin was first described in the early 1990s as a component of a mammalian cell adhesion complex. [11] Later it was recognised that the Drosophila protein armadillo which is responsible for mediating the morphogenic effects of Wingless/Wnt is homologous to the mammalian $\beta$-catenin, both in structure as well as in function. ${ }^{[12]}$

In 2004, Hsien-Chang Shih et al. used immunohistochemical methods for analyzing the expression of E-cadherin and beta-catenin in normal and malignant endometrium. ${ }^{\text {[7] }}$ They proposed that in the normal endometrium, E-cadherin and cytoplasmic beta-catenin expression in glandular cells was higher in the proliferative phase as compared to the secretory phase. In endometrial carcinomas, the expression of E-cadherin and cytoplasmic beta-catenin is decreased, more so in higher grade tumors. Nuclear expression of beta-catenin was observed in the glandular cells in high-grade endometrial cancer. In our study, all cases of proliferative endometrium showed strong $(2+)$ membrane and cytoplasmic positivity for beta-catenin in the endometrial glandular cells. However 20\% cases of atypical endometrial hyperplasia and $46 \%$ of cases of endometrial carcinoma showed nuclear localization of beta-catenin. Thus, our findings were concordant with the findings of Hsien-Chang Shih et al.

Gema Moreno-Bueno et al. studied the alterations in APCbeta catenin pathway in endometrial carcinogenesis. They found a statistically significant association between betacatenin nuclear expression and the endometrioid phenotype $(\mathrm{P}=0.002)$. In their study nuclear beta-catenin expression was found in $31.2 \%$ of endometrioid endometrial carcinomas (EECs) and in 3\% of non endometrioid endometrial carcinomas (NEECs). ${ }^{[8]}$ However, in our current study, $42 \%$ of endometrioid carcinomas and both the cases of serous carcinomas showed nuclear localization of beta-catenin. Gema Moreno-Bueno et al. further demonstrated high prevalence of molecular alterations of the APC/beta-catenin pathway, but only mutations in betacatenin gene correlated with aberrant nuclear localization of beta-catenin. We did not do molecular analysis and our findings of beta-catenin alterations were based on immunohistochemistry alone.

Xiong $\mathrm{Y}$ et al. studied the expression of beta-catenin, Glut-1, and PTEN proteins in uterine endometrioid adenocarcinoma and its precursor lesions. Abnormal (marked membranous/cytoplasmic, cytoplasmic and/or nuclear or negative) expression rates of beta-catenin in EIN lesions $(50 \%, 12 / 24)$ and endometrioid adenocarcinoma $(66.7 \%, 16 / 24)$ were significantly higher than that of benign hyperplasia $(10.2 \%, 6 / 59)$ respectively $(\mathrm{P}<0.01)$. [9] In our study, it was found that atypical hyperplasia and endometrial carcinoma showed weaker membrane positivity and nuclear expression compared to proliferative endometrium and hyperplasia without atypia.

Saegusa $\mathrm{M}$ et al. investigated the role of aberrant betacatenin expression during endometrial tumorigenesis. [13] Cell membrane $\beta$-catenin immunoreactivity showed a sequential diminution from normal, through atypical hyperplasia, to grade 3 carcinoma. In contrast, the nuclear accumulation in atypical hyperplasia and grade 1 or 2 tumor was higher than in hyperplasia without atypia. In our study we found similar results.

\section{Conclusion}

Normal expression of beta-catenin in endometrial glandular cells during proliferative phase of menstrual cycle is on membrane and in cytoplasm. The present study reinforces that nuclear expression of beta-catenin strongly correlates with endometrial pathology, namely, hyperplasia and carcinoma. It has been observed that there was markedly increased nuclear expression, reduced and weaker membranous-cytoplasmic expression with increasing histological grade of endometrial carcinoma. Beta catenin can therefore be used as a surrogate maker in early diagnosis of precancerous lesions and also as an important prognostic marker in patients with endometrial carcinoma.

\section{Acknowledgements}

We are indebted to the faculties as well as technical staff of the Department of Pathology, Nilratan Sircar Medical College and Hospital for their constant support and motivation during the execution of this study.

\section{References}

1. Kurman RJ, Ellenson LH, Ronnett BM. Precursor Lesions of Endometrial Carcinoma. In: Kurman RJ, editor. Blaustein's Pathology of the Female Genital Tract. Sixth Edition. New York: Springer; 2011:360.

2. Okuda T, Sekizawa A, Purwosunu Y et al. Genetics of Endometrial Cancers. Hindawi Publishing Corporation Obstetrics and Gynecology International. 2010; 2010: 8 pages.

3. Hendrickson MR, Longacre TA, Kempson RL. Female Reproductive System and Peritoneum. In: Sternberg's Diagnostic Surgical Pathology. Fifth Edition. Philadelphia: Wolters Kluwer Health; 2015. 2204.

4. Jiang WG. E-cadherin and its associated protein catenins, cancer invasion and metastasis. Br J Surg. 1996; 83. 437-46.

5. Gumbiner BM. Cell adhesion: The Molecular Basis of Tissue Architecture and Morphogenesis. Cell. 1996; 84. 345-57. 
6. Provost E, Rimm DL. Controversies at the cytoplasmic face of the cadherin-based adhesion complex. Curr Opin Cell Biol. 1999; 11 (5). 567-72.

7. Shih HC, Shiozawa $\mathrm{T}$, Miyamoto $\mathrm{T}$ et al. Immunohistochemical Expression of E-cadherin and betacatenin in the Normal and Malignant Human Endometrium: An Inverse Correlation between E-cadherin and Nuclear beta-catenin Expression. Anticancer Research. 2004; 24. 3843-50.

8. Bueno GM, Hardisson D, Sanchez C, Sarrio D, Cassia R, Prat J. Abnormalities of the APC/beta-catenin pathway in endometrial cancer. Oncogene. 2002; 21.7981 - 90.

9. Xiong Y, Xiong YY, Zhou YF. Expression of betacatenin, Glut-1, PTEN proteins in uterine endometrioid adenocarcinoma and its precursor lesions. Zhonghua Bing Li Xue Za Zhi. 2009; 38(9). 594-9.

10. Scholten AN, Creutzberg CL, van den Broek L, Noordijk EM, Smit VT. Nuclear beta-catenin is a molecular feature of type I endometrial carcinoma. J Pathol. 2003; 201(3). 460-5.

11. Mc Crea PD, Turck CW, Gumbiner B. A homolog of the armadillo protein in Drosophila (plakoglobin) associated with E-cadherin. Science. 1991; 254 (5036). 1359-61.

12. Kemler R. From cadherins to catenins: cytoplasmic protein interactions and regulation of cell adhesion. Trends Genet. 1993; 9 (9). 317-21.

13. Saegusa M, Hashimura M, Yoshida T, Okayasu I. Betacatenin mutations and aberrant nuclear expression during endometrial tumorigenesis. Br J Cancer. 2001; 84. 209-17.

*Corresponding author:

Dr Sagarika Sarkar, Flat No. 302, Sri Sai Ram Kalyan Kelsey, 27th Cross, Ejipura, P.O.: Vivek Nagar, Bangalore: 560047, India

Phone: +910801770103

Email: sagarika0507@gmail.com

Financial or other Competing Interests: None. 